\title{
Designing Musical Soundtracks for Brain Controlled Interface (BCI) Systems
}

\author{
Richard Ramchurn \\ School of Computer Science \\ University of Nottungham \\ Nottingham \\ UK \\ richard.ramchurn@nottingham.ac.uk
}

\author{
Alan Chamberlian \\ School of Computer Science \\ University of Nottungham \\ Nottingham \\ UK \\ alan.chamberlian@nottingham.ac.uk
}

\author{
Steve Benford \\ School of Computer Science \\ University of Nottungham \\ Nottingham \\ UK \\ steve.benford@nottingham.ac.uk
}

\begin{abstract}
This paper presents research based on the creation and development of two Brain Controlled Interface (BCI) based film experiences. The focus of this research is primarily on the audio in the films; the way that the overall experiences were designed, the ways in which the soundtracks were specifically developed for the experiences and the ways in which the audience perceived the use of the soundtrack in the film. Unlike traditional soundtracks the adaptive nature of the audio means that there are multiple parts that can be interacted with and combined at specific moments. The design of such adaptive audio systems is something that is yet to be fully understood and this paper goes someway to presenting our initial findings. We think that this research will be of interest and excite the Audio-HCI community.
\end{abstract}

\section{ACM Reference format:}

Richard Ramchurn, Alan Chamberlain \& Steve Benford. Designing Musical Soundtracks for Brain Controlled Interface (BCI) Systems. In Proceedings of Audio Mostly'18, September 12-14, 2018, Wrexham, United Kingdom, 2018 (AM'18), 8 pages. https://doi.org/10.1145/3243274.3243288 .

\section{CCS Concepts}

- Human Centered Computing $\rightarrow$ Collaborative and social computing; Collaborative and social computing theory, concepts and paradigms; Social content sharing; Collaborative content creation.

\section{KEYWORDS}

Brain Controlled Interface (BCI), Music, Audio, Design, Composition, HCI, Creativity, Audience, Film, Art, New Media

\section{Introduction}

To initially frame the research presented in this paper we start by examining the first application of a brain computer interface (BCI), Alvin Lucier's Music for the Solo performer in 1964 [20], which was the sonification of raw EEG (ElectroEncephaloGraphical) data via percussive instruments. Using EEG data to create musical experiences is not new, it is possibly the most common application of BCIs in the arts. This could be because of its precedence, the similarity of EEG signals to audio frequencies, the quality of artists to adopt new technologies, probably a combination of all these things. Indeed, as music can be a way to express an internal state, it makes sense to utilise the flow of data from the brain, this makes the use of EEG signals ripe for musical experimentation, exploration and expression. Recently we have witnessed a growth in in the development and use of musical BCIs as artists have started to gain access to consumer grade headsets.

As more projects, tools and performances are developed, the 'back catalogue' of musical BCI research has grown, building a body of knowledge and understanding gained from this research. This in turn has led to the emergence of a set of common mechanisms [23], for example the passive method whereby brain signals are used in an unconscious manor; the user does not have to learn how to control their brain signals and the material they interact with is preprogrammed. We expand upon the notion of passive interaction later in this paper.

Some artistic applications of BCIs in sound and music have been integrated into larger sensory experiences [21]. When used in film we experience music as part of a range of sensory inputs, the music and sound inform how we feel and understand the narrative of the film, it adds to the picture and it is taught in film school that sound is more important than the image [4]. Just as the EEG waves can be sonified, similar techniques may be applied to visualise data. By combining the audio and visual aspects of such representations (and applications of EEG data) it may be possible to develop BCI-based tools that can support and present mixed-media representations. This then enables the development of interactive narratives, such as those used in film, which bring together audio and images to create a unified experience - for both audience and creator.

Some of the most challenging problems that one faces in the realm of interactive cinema relate to; the development and production of the large amount of media necessary to create such an experience
Permission to make digital or hard copies of all or part of this work for personal or classroom use is granted without fee provided that copies are not made or distributed for profit or commercial advantage and that copies bear this notice and the full citation on the first page. Copyrights for components of this work owned by others than ACM must be honored. Abstracting with credit is permitted. To copy otherwise, or republish, to post on servers or to redistribute to lists, requires prior specific permission and/or a fee.

Request permissions from Permissions@acm.org.

AM'18, September 12-14, 2018, Wrexham, United Kingdom

(C) 2018 Association for Computing Machinery.

ACM ISBN 978-1-4503-6609-0/18/09 ...\$15.00

https://doi.org/10.1145/3243274.3243288 
[15], the spilt in attention of the user between the film reception and the interactivity, where the active action of making a decision, and physically acting on that breaks the narrative immersion of the viewer [3] and finally, preserving the flow of the cinematic experience when such interactivity occurs [22]. We will discuss how we addressed these problems in design and practice.

\section{Overview of the work}

The focus of or our research is on interactive cinematic experiences. Thus far we have created two films which have been professionally released, these enable the users (of the experiences) to interact via EEG brain data and create narrative experiences that are adaptive, this is to say, they adapt to the individual user's data and are unique on each viewing, yet still preserve a narrative coherence that one would expect from a film-driven experience. The creation of a cinematic narrative in film involves many interacting disciplines, in this research we focus on the creation of the soundtrack and the audio mix, how it was created, how it was developed/designed, the approaches and reasoning made to make it work, and what opportunities interactive sound design can present that may solve some of the known problems inherent in new interactive cinematic experiences, such as the ones that we will describe shortly.

Thus far we have produced and professionally released two interactive films. In this section we describe each film, our research aims, give a synopsis of the film and the content that we used. "The Disadvantages of Time Travel"1 (DOTT) an expressionistic tale of an adolescence spent balanced between reality and fantasy which attempts to immerse the interactor in the psychology of the main character. The second film "The MOMENT"2 , a sci-fi thriller noir pulls on traditional cinematic techniques to tell its story. Both films are constructed on the fly; that is the second by second edit, music and sound design are defined in real-time by the interactors live brain data. In this paper we focus more on our most recent film, The MOMENT.

Earlier in the paper we briefly touched on the notion of Passive Interaction [23], but what is it, why use it with audio as part of a BCI system and why might it work as part of an artistic experience? Passive interaction is defined as having no direct conscious input, in this case data is monitored from the brain and this can be used as a way to interact and effect media. The changes that occur can be fed back to a user or audience through sound, music moving images and narrative rhythms. This mechanism has also been categorized as an affective BCI [1]. Our systems used the afore-mentioned passive control mechanism, as opposed to what is known as a direct, or selective method [23]. There were two key reasons for this; firstly, training is essential if one is to use a BCI direct control mechanism and it is not practical for the interactors (users) to go through a lengthy training period in a public installation setting and secondly, and importantly for this this type of experience, there is evidence that a conscious control can break narrative tension [18]. This would prove ineffectual for anyone trying to develop an 'interesting' narrative, either using visuals or audio.

${ }^{1}$ 2015. Received worldwide media attention during its UK tour https://www.fact.co.uk/projects/the-performing-data-project/albinomosquito-thedisadvantages-of-time-travel.aspx https://www.imdb.com/title/tt8072006

\subsection{Addressing the Problems}

One of the first things that people are taught in film school is how different music can change the reception of a scene. Just imagine watching a horror film with the soundtrack from a comedy [5], it might be amusing, but it certainly won't convey the meaning that the director wants the audience to understand. In our productions creating more music was more economically viable than having to film multiple scenes. The second issue that arises when one interacts with an interactive film concerns an incompatibility between the experiential flow inherent in watching a movie, which gives the audience a sense of continuity from start to finish, and the break and shift in agency of the viewer that is usually required for interaction to occur.

The interactive properties of BCI mean it is a tool that is perfect for interaction where continuity needs to be undisrupted, by using BCI the interactor does not need to stop the film to interact, in fact by using the passive mode of control as earlier described she doesn't even need to be aware that she is steering/controlling the film, its soundtrack and edits. By making these interactive moments ubiquitous, they can happen at any time - we have found that the problem of split attention is reduced, and by mixing the music and sound design in predefined combinations the flow of the film is preserved.

\section{Designing the Systems}

In this section we give an overview of the structure of the experiences that we designed and developed. By using graphical representations of the way that the instruments were aligned to audio and video, and the media structure of the experience we are able to demonstrate the way that the experiences worked with media as part of a BCI system. What follows is an overview of how each of the two films selected and mixed pre-composed music, what the design justifications were and how they related to the experience.

\section{The Disadvantages of Time Travel}

The BCI hardware used in this experience was a Neurosky Mindwave headset ${ }^{3}$. It was chosen for its ease to fit, important when working with the public. It has pre-defined algorithms that output data based on attention and meditation, in this we used the latter and mapped it to the soundtrack mix, which we shall now explain. For each scene in the film a piece of music was composed, this was broken down into at least four sub-mixes, typically percussion, bass, synths, guitar. We then mapped the meditation levels, (a number between 0 and 100) to the mix of these stems.

\footnotetext{
2 2018. Premiered at Sheffield Doc Fest and due to tour UK 2018-19. https://www.imdb.com/title/tt7853742/?ref_=nm_knf_i2

${ }^{3}$ Neurosky MindWave Mobile headset http://neurosky.com/
} 


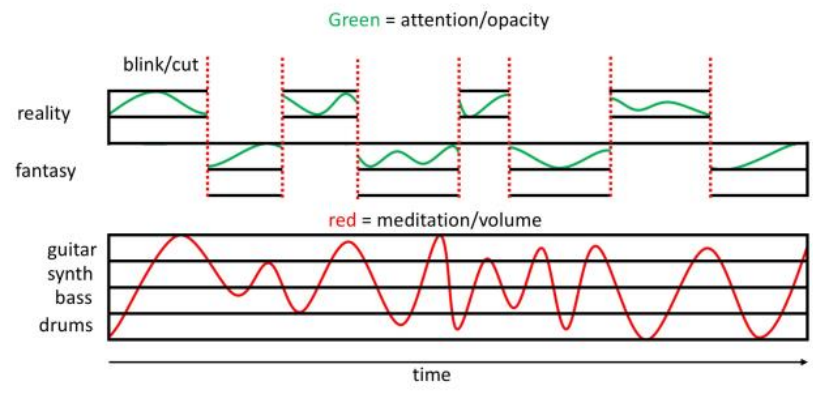

Figure 1. The mapping brain data to audio and video in The Disadvantages of Time Travel

This meant that when a user watched the film and the meditation signal was equivalent to 0 there would be no music, however between 0 and 25 the drum stem would play, between 25 and 50 a bass would be introduced, between 50 and 75 the user would hear the synths in the mix and between 75 and 100 the full mix including guitar could be heard. The motivation behind this mapping was that the higher the level of Meditation the fuller the music would appear, (see Figure 1. above). The system was originally built with a python code sending the headset data via Open Sound Control (OSC) to a quartz composer patch, which was later re-designed in MAX/MSP (a visual programming package). As previously mentioned the artistic aim of this piece was to put the viewer in the same dissonant phycological state as the main character as he moved between reality and fantasy and so the interactors' own levels of meditation would be reflected back to the viewer as a louder, fuller composition.

\section{The MOMENT}

Again, we used the Neurosky Mindset headset in this piece, but for this experience we shifted from mapping data directedly to an audio or visual attribute and designed a new interactive approach Following the Performance Led Research in the Wild [2] approach this design was iteratively refined by previous studies [18] to use the feedback attribute of the filmic cut and visual sensitive Attention data from the Neurosky headset [14]. It was also guided by the theory of event segmentation [26]. The technical aim of this film was to recreate common recognizable cinematic techniques to make a film that was familiar in its form. In order to accomplish this, we used momentary drops in attention data (as sensed from the EEG data) to produce cuts between two of three parallel narrative threads which made up each interactive scene (see Figure 2.). Each thread has two musical treatments, the primary that is always on and the secondary which fades in and out as the secondary thread cuts (see Figure 3.). The characteristics of the treatments were defined by how they would appear in the constructed film, the primary treatment included rhythms and melodies, musical elements and foley (environmental sound effects) and a secondary treatment of the scene which consisted of drones and atmospheric soundscapes. The dialogue was part of the video files themselves and is always on.
The system was designed so that in each scene one thread is designated the primary and the other secondary. When playing, the secondary music fades in and out with the secondary visual scenes. At the end of each interactive scene the primary / secondary viewing ration and the frequency of cuts is used to decide on the next narrative thread combination. This was also built in MAX/MSP.

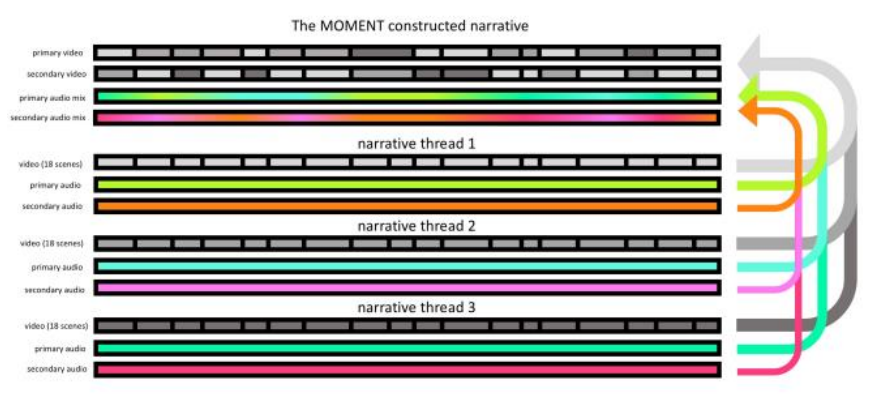

Figure 2. the audio and video media structure of The MOMENT

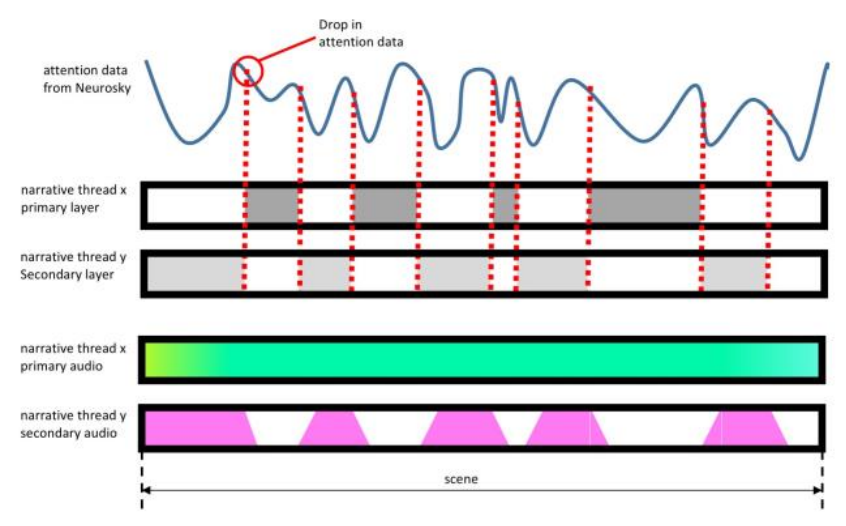

Figure 3. Mapping of attention drops to audio and video in The MOMENT

\section{Musicians, Compositions, Considerations and Processes}

In order to fully understand and appreciate the process of composing and designing the soundtrack of The MOMENT we gather first-hand accounts of the activity of creation. It is important to note that the composers and the director all worked remotely from each other, across different countries. Firstly, we have kept a $\log$ of all the online discussions that took place between the artist/director and the two composers, these discussions occurred over the length of the project and are an important record of the design and developments of the dynamic soundtrack for the film. Secondly, we were able to carry out interviews with the composers so that we could fully understand the motivations and reasoning behind their compositions and the approaches that they employed. 
The insights from both the conversational logs and the interviews provide an important resource that can inform the design of other adaptive experiences and are able to enable us to look at the way that soundtracks for such experiences are seen by composers, we briefly present some insights from the composers here. It is clear that unlike traditional soundtracks (which the authors have experience of composing) that adaptive BCI-based soundtracks need to be carefully designed and an approach taken that can account for fluctuations in EEG data, and as we have earlier discussed in relation to the visual attributes of a film, the continuity of the audio tracks of the film also need to be perceived as continuous. This continuity needs to be applied both to the flow of the audio, and in respect to its relation to the visual elements of the film. A break in continuity can indirectly impact upon the EEG response of the viewer and therefore the viewing experience. In using BCI-based systems to interface with content, both the composer and viewer are moving beyond the standard HCI notions of what usability is and into a realm of design where poor design and disruption can lead to an unusable experience. We further expand upon audience responses and perceptions later in the paper but in this section, we focus on the composers and director.

Composer 1 worked on both on The Disadvantages of Time Travel and The Moment. He experiments and performs with controllable feedback instruments the he designs and produces himself and has scored feature films, dance pieces, and pieces for choir. He holds several degrees in composition.

Composer 2 has collaborated with the director for over 10 years, he is self-taught, and he makes his own hardware, synths and circuit bent instruments. He plays on his own and with well know bands at festivals. He has been making music for over 20 years and disseminates his craft by running workshops in across the UK and across Europe.

The composers' strategy was to support the mechanisms of interaction as part of the BCI-system used in the experiences. One of the mechanisms employed avoided the need for the adherence to musical key across the pieces. With Composer 1 (C1) writing what they called a 'traditional' piece of music for the primary layer and a 'soundscape' for the secondary and Composer 2 (C2) developing a piece using modular synths. $\mathrm{C} 1$ was particularly interested in developing a piece that had a semi 'aleatoric' (random) nature in which the user became a type of $\mathrm{C} 2$ "conductor". C2 states, "we started sharing tempos and keys for each cut to match beat layers and rhythms". C2 "storyboard and description of the vibe for the scene was well useful, we had a meeting online to discuss ideas for the different elements and segments, it took me good few months to get my head round how it would work exactly, it's only when we started to get the video rushes in for each section you could then see how it fits with the other threads of the movie and how they connect and interact".

In order to see if their work fitted together the composers used a strategy of sharing 'rough mixes' to each other in order that they could understand what each of the composers was working on. The compositional work was divided between the composers, with each composer basing their compositions around 3 characters. C2 "it started getting more difficult to arrange when the secondary tracks were to be made and how they would fit with the primaries. Sometimes a sound clash would work between the styles and others it needed to be replaced or tweaked to fit so lots of edits for those threads".

As part of the interview we discussed the notion of a live performance based on the soundtrack, which the composers had already trialled. One theme worthy of note was that that of randomness (see quote below). Both $\mathrm{C} 1$ and $\mathrm{C} 2$ noted that they were interested in, as $\mathrm{C} 1$ stated "aleatoric" composition and had an interest in hearing what this might sound like if things were truly random. In some respects, this is a difficult thing to accomplish, what may at first appear to be random is in fact very structured and controlled. Musical keys are controlled, types of music mixed traditional with a soundscape is managed so as not to cause tonal dissonance and distraction, the whole management and mapping of content is highly organised and controlled within parameters so that the BCI system can work with 'randomness' in this context, which is to all intents and purposes a 'live' real time experience. For discussions relating to this see [6][7]. C2 "It may have to be a case of us choosing tracks for the performance like a setlist, but this wouldn't be brain controlled which I wouldn't want to do. We like the randomness of the whole thing and the fact the audience is essentially running the show and choice without realising is what's inspiring."

\subsection{Modular, non-linear content}

The MOMENT is non-linear in the sense that there is no one definitive path to be taken through the film, it could be more descriptively described as semi-linear. At the end of each scene there is the possibility of 1 of 6 combinations of soundtrack, narrative thread and narrative prominence. However, each time it is watched it is experienced as a linear story, that is there are no chronological discontinuities in the film. The music and sound design have been made and programmed to keep this sense of a linear flow throughout the narrative, utilising audio dissolves between and within scenes, and by keeping a primary scene as having the most detailed foley (the creation of everyday sound effects, usually added to film) and prominent musical elements.

\subsection{Understanding the Audience}

\section{Setting up the Experience}

DOTT was first shown in a caravan which had been converted into a cinema space, this scaled down cinema made for an intimate, immersive space, allowing the sound and images of the film to immerse the viewer/controller in the film and thus more of an impact upon the user's brain data. The next round of screenings was a multi-person version of the film which toured to theatres, auditoriums and screening rooms across the UK. During that tour it was common to be placed in a room that had distractions to the controllers, sometimes a very small audience in a large theatre space, excess light, people working in lighting rigs would cause distractions, all of which was outside the control of the team showing the film. From that experience it became clear that a dedicated screening space was needed, one that was mobile, comfortable and fit the scale of the audiences. Before the film was 
written work began on sourcing and converted a small caravan into a cinema to be used with the up-coming film 'The MOMENT'. The cinema can fit comfortably 7 people and has an improved interior design over the first borrowed caravan. The ceiling mounted projector throws a $2.5 \mathrm{~m}$ screen at a distance of $2 \mathrm{~m}$ from the audience creating a similar field of view as a large cinema auditorium while retaining an intimate space.

The most recent screenings took place at Lakeside Arts in Nottingham and FACT Liverpool. Participants would book their places online either as an audience or controller, the preview screenings were advertised on host venues and the project's websites and social media accounts. The screenings were free to the public and no financial compensation was offered. Upon arrival audience members would be asked to fill in an information and consent form, the interactor was fitted with the Neurosky headset, during which any questions asked about the experience and system were answered. In Liverpool audiences saw the film once then answered a questionnaire and gave an interview. In Nottingham audiences watched the film twice, each time with a different interactor. After each screening they were asked to fill in a questionnaire and were asked to engage in an interview after the final screening. There were 60 viewers, over 21 screenings at FACT over 3 days and 35 viewers over 10 double bill screenings at Lakeside Arts over 2 days. Each film is recorded for a future interactive archive.

Using a BCI interface as an interaction tool is very different from traditional methods of control an audience may have with an interactive narrative. Various methods that have been used in the past include anaglyphic glasses [13] remote controls [24] point and click web interfaces [10] Playstation controllers [19] and apps in cinemas [11] or by themselves [9]. The non-physical and sometimes subconscious interaction that is obtained with a BCI interface has some unique advantages, if integrated into a live playback system. Broadcast media, and cinema is based around technologies that traditionally not support on the fly creation of content. However, as broadcast moves to digital channels and online and cinema is digitised from creation to distribution there are new possibilities inherent to the mediums are being explored $[17,12]$.

When controllers were asked if they may feel a responsibility to make a 'good' film, one that is understood by other audience members they were more concerned that the film could show something weird about their thoughts, that they may share something that others may find strange. Although non-interacting audience members put the responsibility of the quality of the film on the technology or the director. Regardless the interactor creates a unique film, and that person has some form of authorship over the created object. Especially since the film is being recorded.

After viewing the film interactors were curious to know 'how they did' and if the film they made was typical. It was common for people, interactors and audience to prefer the second movie of the double bill, if this is because it 'tied up the pieces' or because it was 'so different the second time' remains to be seen, regardless there is some value in the repeat experience of seeing the film again both for interactors and non-interacting audience.
The choice of sound put alongside a narrative scene meant it could affect the perceived meaning of the whole, for example by adding a build-up of tension, or having a calming soundtrack. As changing the sound behind a scene can change the viewers perception as much as having different visual elements of the film, it changes the meaning and feel of the film. We further explored the users' perceptions of interacting by following up their interactions with interviews. We present some initial findings here, before presenting some questionnaire data.

Users of the experience has sometimes seen other versions of the film and were able to see the differences between the experiences, as seen in the following quotes and were able to see how their version differed from others. We need to further explore if there is a sense of ownership and authorship in regard to such feelings "in a way the movie adapts to your taste, ... I would say it defiantly has its merits but then again would you only want to be presented things that are already interesting to you or would you rather be presented something new that might spark a new interest in you so if you just keep reiterating something that you like then you just end up in your own little bubble and you will never experience anything outside of that or anything original with that you will just be presented and fed with the same kind of thing that you already like ...

"I could feel in my version there were a to more cuts and the voices were little bit different, yeah there was a huge difference"

It was evident from our interviews that some of the users were able to comprehend that they were able to have a direct input on the experience that they were taking part in. One issue that is raised is that of control and the impact of that upon the experience. An issue appears to emerge that relates to the control of the experience and not wanting to have too much of an impact on that, as is seen in the quote below. People were able to see that they could have an impact upon the system. "I was conscious in parts, I'd be dead focused and then id zone out a bit and think oh gosh this relies on my attention ... but then I just tried to tune that out and focus again...I didn't want to flip it too much...I just to really focus on the narrative and get pulled back into the film"

\subsection{Questionnaires}

From the first public viewing of The Moment 33 people responded to our questionnaire. We had previously used a questionnaire-based approach to gather information related to another non-linear system that we had developed [2] and this approach had enable us to further understand the system. By using the questionnaire, we were initially interested in how people perceived the soundtrack and if people could see any correlation between the media elements of the experience. This was important, because as we have mentioned previously, distractions can impact upon the continuity of the narrative, which can negatively impact upon the experience for the user. This would render the experience somewhat intelligible, and in terms of using BCI-based passive approached to interaction and control, a disruption can impact upon the EEG values and therefore have an impact upon the way the edits in the experience can occur. 
In this section we would like to provide some of our findings. We present some responses that have started to lead us to the conclusion that although the viewer noticed a 'relationship', this relationship was not disruptive and was seen as a standard part of the film. Our initial responses suggest that the users understood that there was a correlation between the visual elements of the film and the soundtrack. This is important as it shows us that people were able to understand that there was a relationship between the images and sound.

As we can see from the quotes below, people saw that there was a correlation between the visual aspects of the film and the audio, as one might expect. Interestingly, just taking this handful of examples people appear to draw their own conclusions and reasoned differently about the nature of the relationship between the graphics and the audio. These ranged from changes in 'perspective', 'CGI' effects, graphics changing and people noticing, but not understanding why or how. A further avenue of research that could garner would be to take an ethnomethodological approach (as used in other music research) [16, 8], which would look at the methods and approaches (reasoning) that people use to understand a given context.

"I noticed some of the synth textures changing when the CGI graphics came in and out"

"Sometimes a perspective change changed the sound"

"The sound accompanying the graphics seemed to change when the graphics changed"

"I noticed it change (the music) but couldn't tell you how"

It was apparent that not all of the viewers were fans of the soundtrack, and it's always rather amusing to read responses like the one below when participants respond to questions about 'music'. Such questions make you think about the nature of music and the listeners perception, reception and musical taste.

"Noticed less music and bone shaking bass"

We were also able to see that the soundtrack was an important element of the experience and that it was able to support the users' feeling towards the film and helped them to 'immerse', 'get into' it. This is an important finding and it is something that we need to further research in terms of how BCI-based musical experiences can support immersion.

"How it made me feel (Music) grew in intensity"

"The beats helped me to get into the scene"

Part of the job of the audio was to carry the narrative, by this we mean that the audio (as a separate element) can play continuously without break, while the accompanying edits in the film can happen many times while the soundtrack plays continuously. In respect of the continuity of the narrative both the director of the film and the composers felt that the textual element of the film should not be broken, and that the soundtrack enabled and supported the 'fluid' edits made to the visuals. One of our research questions related to trying to find out if the audience would notice a link between the images, audio and interaction mechanism. Although this paper is not long enough to fully unpack out findings we offer some insights.

\section{$5 \quad$ Future Directions \& Designs}

To continue the research in this field one direction is to scale the experience up, both to maximize the impact of the research and to reach a financially stable model for work of this kind. Currently the film is made interactive by using the brain data of one person at a time, our previous work in engaging 3 people at a time via BCI proved successful where one person was responsible for a specific brain data to filmic technique mapping. However filmic techniques are finite, current brain scanners are expensive at scale (although not prohibited so) and come with their own technical challenges.

Another way to increase the audience engagement is to make the individual experiences that are produced from the interaction of the system by a given user available to be seen again via an online archive. The archive would include every recording of the film, as well as meta data such as number of cuts and route taken, which could be used as an academic resource. As we noticed in the exit interviews there is a demand for this, the audience are interested in how they 'compared' to others and by making the archive interactive users can see where their film sits in the corpus.

Although we have made great effort to recreate a small-scale cinema that does not mean The MOMENT or even DOTT can't be experienced in a larger venue. There is a resurgence of the live score or re-scoring of old films, and audiences exist that enjoy the experience live music in such contexts. Taking this into account designing a double bill that is live-scored by our musicians could be an interesting tour. There are also certain design contributions to be discovered in making such a performance system possible as the musicians' Ableton projects will have to be linked to the BCI system to serve them with timely project media. This also brings us back to the notion of the interactor being the 'conductor' of such a performance.

Moving away from the possibilities of increasing reach of the research and experiences to improving the system itself, we could look at using improved methods of data capture, there are many different methods of translating neural activity to data, at higher resolution, accuracy and bandwidth. By using a medical grade EEG, fNIRS, or fMRI and the collaboration with neuroscientists one could design engaging forms of neuro therapy, that have an element of creativity and entertainment as part of the system.

In terms of the design of a future film it may be possible to examine different types of narrative. In The MOMENT we used some genre tropes of the thriller, the hero's journey and science fiction that audiences are primed to receive and understand. Since the medium of brain-controlled film offers new possibilities in narrative not open to traditional linier film there could be more to discover in the way stories can be told and integrate that into the design of a new film. 


\section{Conclusions}

Brain Controlled Interfaces offer new and exciting interactional possibilities for both the producer and consumer of music and mixed media experiences. In order to fully appreciate and use such systems we need to be aware of the ways in which such experiences need to be designed from the design of the environment, through to the way the content (audio \& visual) is put together in order to deliver continuity and a narrative arc. In this paper we have specifically focused on the development of the soundtrack, as we think that this will be of interest to the Audio Mostly community. It is clear from our research that there are different approaches that need to be taken in order to specifically develop and deliver BCIbased experiences in which audio plays a significant part. The ways in which the audio can hold the narrative together are particularly important from the perspective of the director and composer, from our questionnaire responses it was evident that the audience understood that there was a relationship between the audio and visual elements of the film. The idea of the audience as conductor as raised by one of the composers is something that we look to further understand and develop as our project evolves and we start to develop software tools that will enable more composers and musicians to create and perform for BCI-based experiences.

\section{ACKNOWLEDGMENTS}

This work was supported by the Engineering and Physical Sciences Research Council [grant number EP/L019981/1] project Fusing Semantic and Audio Technologies for Intelligent Music Production and Consumption.

\section{REFERENCES}

[1] Marvin Andujar, Chris S. Crawford, Anton Nijholt, France Jackson, and Juan E. Gilbert. 2015. Artistic brain-computer interfaces: the expression and stimulation of the user's affective state. Brain-Computer Interfaces 2, 2-3: 60-69. https://doi.org/10.1080/2326263X.2015.1104613

[2] Steve Benford, Chris Greenhalgh, Adrian Hazzard, Alan Chamberlain, Maria Kallionpää, David M. Weigl, Kevin R. Page and Mengdie Lin (2018) "Designing the Audience Journey through Repeated Experiences" In Proceedings of ACM CHI 2018 - Conference on Human Factors in Computing Systems. Montréal, Canada. April 21st - 26th 2018. DOI - 10.1145/3173574.3174142

[3] Nitzan Ben-Shaul. 2008. Hyper-Narrative Interactive Cinema. New York.

[4] David Bordwell and Kristin Thompson. 2001. Film Art: An Introduction. https://doi.org/film;cultural studies;semiotik

[5] Alan Chamberlain and Adrian Hazard (2015) Sonifying the Scene: re-framing and manipulating meaning through audio augmentation. In: DMRN+10: Digital Music Research Network, December 2015, London

[6] Alan Chamberlain (2017) "Are the Robots Coming? Designing for Autonomy \& Control in Musical Creativity \& Performance." Audio Mostly 2017: Augmented and Participatory Sound/Music Experiences, 23-26 August. Queen Mary University of London (London, UK) ACM DOI https://doi.org/10.1145/3123514.3123568
[7] Alan Chamberlain, Mads Bødker, Adrian Hazzard, David McGookin, David De Roure, Pip Willcox and Konstantinos Papangelis (2017) “Audio Technology and Mobile Human Computer Interaction: From Space and Place, to Social Media, Music, Composition and Creation", In the International Journal of Mobile Human Computer Interaction (IJMHCI) Volume 9, Issue 4, October - December 2017 pp. 25 - 40. DOI https://doi.org/10.4018/ijmhci.2017100103

[8] Alan Chamberlain and Andy Crabtree (2016) "Searching for music: understanding the discovery, acquisition, processing and organization of music in a domestic setting for design". in Personal and Ubiquitous Computing. pp. 1-13. Springer, ISSN 1617-4917, DOI: 10.1007/s00779-016-0911-2

[9] CtrlMovie Home - CtrlMovie. Retrieved May 30, 2018 from http://www.ctrlmovie.com/

[10] Jonathan (University of York) Hook. 2018. Facts , Interactivity and Videotape : Exploring the Design Space of Data in Interactive Video Storytelling. In Proceedings of the ACM TVX International Conference on I nteractive Experiences for Television and Online Video. ACM TVX'18 International Conference on Interactive Experiences for Television and Online Video.

[11] William Hughes. Fox's Choose Your Own Adventure movie will let audiences choose which awful things happen to these idiot kids. Retrieved May 28, 2018 from https://news.avclub.com/foxs-choose-your-own-adventuremovie-will-let-audiences-1825580340

[12] Interactive content on Netflix. Retrieved May 30, 2018 from https://help.netflix.com/en/node/62526

[13] J M King. 2008. Stereoscopic cinema \& the origins of 3-D film, 1838-1952. The University Press of Kentucky. Retrieved from http://search.proquest.com/docview/225718657?accountid=1 5533

[14] Youngrae Kim, Jinyoung Moon, Hyung-Jik Lee, ChangSeok Bae, Sungwon Sohn. 2012. Integration of electroencephalography based services into consumer electronics. IEEE 16th International Symposium. https://doi.org/10.1109/ISCE.2012.6241692

[15] Lev Manovich. 2001. The Language of New Media. Screen 27, 1: 354. https://doi.org/10.1386/nl.5.1.25/1

[16] Glenn McGarry, Peter Tolmie, Steve Benford, Chris Greenhalgh and Alan Chamberlain (2017) "They're all going out to something weird: Workflow, Legacy and Metadata in the Music Production Process.“ ACM CSCW 2017. DOI 10.1145/2998181.2998325

[17] Object-Based Media - BBC R\&amp;D. Retrieved May 30, 2018 from https://www.bbc.co.uk/rd/object-based-media

[18] Matthew Pike, Richard Ramchurn, Steve Benford, and Max L. Wilson. 2016. \#Scanners. In Proceedings of the 2016 CHI Conference on Human Factors in Computing Systems - CHI '16, 5385-5396. https://doi.org/10.1145/2858036.2858276

[19] Quantic Dream. Retrieved May 28, 2018 from http://www.quanticdream.com/en/

[20] Volker Straebel and Wilm Thoben. 2014. Alvin Lucier's Music for Solo Performer: Experimental music beyond sonification. Organised Sound 19, 01: 17-29. https://doi.org/10.1017/S135577181300037X 
[21] Staalhemel | responsive environment for brainwaves. Retrieved March 7, 2016 from http://www.staalhemel.com/

[22] Pia Tikka. 2010. Enactive media - generalizing from enactive cinema. Digital Creativity 21, 4: 205-214. https://doi.org/10.1080/14626268.2011.550028

[23] Amy Wadeson, Anton Nijholt, and Chang S. Nam. 2015. Artistic brain-computer interfaces: state-of-the-art control mechanisms. Brain-Computer Interfaces 2, 2-3: 70-75. https://doi.org/10.1080/2326263X.2015.1103155

[24] Ian Willoughby. Groundbreaking Czechoslovak interactive film system revived 40 years later | Radio Prague. Retrieved May 28, 2018 from http://www.radio.cz/en/section/panorama/groundbreakingczechoslovak-interactive-film-system-revived-40-years-later

[25] Jeffrey M. Zacks and Khena M. Swallow. 2007. Event Segmentation. Current Directions in Psychological Science. Vol 16, Issue 2, pp. 80 - 84. 2007

[26] Jeffrey M. Zacks and Khena M. Swallow. 2007. Event Segmentation. Current Directions in Psychological Science. Vol 16, Issue 2, pp. 80 - 84. https://doi.org/10.1111/j.1467$\underline{8721.2007 .00480 . \mathrm{x}}$ 